\title{
Association study between C7673T polymorphism in apolipoprotein B gene and cerebral infarction with family history in a Chinese population
}

\author{
Le Zhang, Yi Zeng, Mingming Ma, Qidong Yang, Zhongyang Hu, Xiaoping Du \\ Institute of Neurology, Xiangya Hospital, Central South University, 87 Xiangya Road, Changsha, Hunan - 410 008, \\ People's Republic of China
}

\author{
Address for correspondence: \\ Dr. Le Zhang, \\ Institute of Neurology, Xiangya \\ Hospital, Central South University, \\ 87 Xiangya Road, Changsha, \\ Hunan - 410008 , \\ People's Republic of China. \\ E-mail:macklon12@yahoo.com.cn
}

PMID: 19934556

DOI: $10.4103 / 0028-3886.57805$

\begin{abstract}
Background: Apolipoprotein B (ApoB) levels have been shown to be associate with risk of ischemic stroke. The apolipoprotein $B$ gene (APOB) polymorphisms may influence levels of $A p o B$ and risk of ischemic stroke, but whether they are associated with risk of ischemic cerebral infarction (CI) with family history (CIFH) or not is unknown. Aims: To investigate the possible association of the polymorphism of APOB C7673T with CIFH in Han Chinese from Changsha. Settings and Design: The study population included 47 patients with CIFH and 83 patients with cerebral infarction with no family histroy (CINFH). Control population included 100 healthy subjects with no history of ischemic stroke matched for age, sex, and ethnic background. Materials and Methods: The APOB C7673T polymorphism was analyzed by the polymerase chain reaction-restriction fragment length polymorphism (PCR-RFLP). Plasma lipids levels were also examined by standard enzymatic methods and enzyme-linked immunosorbent assay (ELISA). Statistics: The statistical analysis was done using SPSS 14.0 software package. A $P$ value $<0.05$ was taken as significant. Results: This study showed an association between the APOB C7673T polymorphism and $\mathrm{Cl}$, especially $\mathrm{CIFH}$ in Han Chinese from Changsha. The T alleles freeuency of the C7673T polymorphism was significantly higher in both the CIFH and CINFH groups when compared with the control group $(P<0.01 ; P<0.05$; respectively). And the $\mathrm{T}$ allele frequency in $\mathrm{CIFH}$ group $(0.17)$ was higher than CINFH (0.09) group $(P=0.056)$. In both the CIFH and CINFH groups the serum levels of TC and LDL cholesterol were significantly high in the TT and TC genotypes than that in the CC genotype $(P<0.01)$, while the serum level of HDL cholesterol in the TT and TC genotypes was significantly lower than that in the CC genotype $(P<0.05)$. Conclusions: The APOB C7673T polymorphism is related to $\mathrm{Cl}$, especially $\mathrm{CIFH}$ through changing serum lipid levels in a Chinese population.
\end{abstract}

Key words: Apolipoprotein B gene, C7673T polymorphism, cerebral infarction, family history

\section{Introduction}

Ischemic stroke (IS) is the leading cause of mortality and disability in China, and in about $78 \%$ of ISs the mechanism is atherothrombotic. ${ }^{[1]}$ There is significant familial aggregation in the occurrence of atherothrombotic IS. ${ }^{[2]}$ The aetiology of IS is heterogeneous and the recent studies emphasises 
the contribution of genetic as well as environmental factors. ${ }^{[3]}$ Although no gene has been convincingly shown to be of importance for cerebral ischemia $(\mathrm{CI})$, the obvious candidate genes are those with an established or potential role in atherosclerosis. ${ }^{[4]}$

Apolipoprotein B (ApoB) plays a central role in lipid transport throughout the body. In addition to its lipid-transport function, ApoB is the major atherogenic apolipoprotein in humans and experimental animals. ${ }^{[5]}$ The gene coding for ApoB is polymorphic, and it has been reported this genetically determined variation has a close relationship with the pathogenesis of atherosclerosis. ${ }^{[6]}$ Although apolipoprotein B (APOB) C7673T polymorphism is not involved in amino acid change, it was reported to play an important role in the pathogenesis of atherosclerosis coronary heart disease and myocardial infarction. ${ }^{[7]}$

Our previous study has indicated that APOB C7673T polymorphism is associated with cerebral hemorrhage in Changsha Han Chinese. ${ }^{[8]}$ In this study we, investigated the importance of APOB C7673T polymorphism as a risk factor for cerebral infarction with family history (CIFH) and also its relationship with serum lipid levels in the Chinese population.

\section{Materials and Methods}

This was a case-control study conducted in the Department of Neurology, Xiangya Hospital, Changsha, Hunan, People's Republic of China from January 2005 to October 2008. All subjects enrolled in the study were Han Chinese. The diagnosis of CI was established by clinical evaluation and brain magnetic resonance imaging (MRI). The diagnostic criteria were based on the World Health Organization International Classification of Diseases, $9^{\text {th }}$ Revision, codes 430-438. Patients with CIFH were defined as patients with at least one first degree relative with IS. The study population consisted of 47 patients with CIFH ( 25 males and 22 females; mean age, $53.48 \pm 10.22$ years) and 83 patients with cerebral infarction without family history (CINFH) (45 males and 38 females; mean age, $58.62 \pm 10.37$ years). The contols included 100 healthy subjects with no history of CI matched for age, sex and ethenic group (53 males and 47 females; mean age, $53.82 \pm 13.26$ years) and were randomly recurited from healthy individual of the hospital. The healthy controls were genotyped in our previous study ${ }^{[8]}$ Patients with cardioembolic CI and $\mathrm{CI}$ due to arteritis, trauma, hemotological diseases, and drugs were excluded. Other patients excluded from the study included patients with liver disease, renal disease, autoimmune diseases, pregnant women and also patients on lipid-lowering therapy within the previous five years.
Detailed information on vascular risk factors: Sex, age, body mass index (BMI), hypertension, diabetes mellitus, ischemic heart disease, smoking history (defines as current, past, or never), and medication use (particularly antihypertensive drug and lipid-lowering agents) was obtained from each subject at baseline. BMI was calculated by the formula, weight divided by square root of the height $\left(\mathrm{kg} / \mathrm{m}^{2}\right)$ and hypertension was defined as blood pressure $\geq 140 / 90 \mathrm{mmHg}$ measured on two separate occasions and/or the use of antihypertensive medication. All the subjects had their fasting serum total cholesterol (TC), triglyceride (TG), and high density lipoprotein-cholesterol (HDL-C), low density lipoprotein-cholesterol (LDL-C), ApoAI, ApoB100 and lipoprotein(a) on the day of the event. Serum levels of TC, HDL, and TG were measured by standard enzymatic methods. LDL cholesterol levels were calculated with the Friedewald formula (LDL $=$ TC- HDL-C-TG/5). If both, total cholesterol and triglyceride levels, are elevated then a modified formula was used: LDL-C $=$ TC-HDL-C- $(0.16 \times$ TG), which provided an approximation with fair accuracy for most people. LP (a) levels were measured by ELISA. The levels of ApoB100 and ApoAI were detected by immunoturbidimetric assay.

Genomic DNA was extracted from peripheral blood leukocytes in all the patients and controls using proteinase $\mathrm{K}$ and the phenol/chloroform method. The determination of APOB C7673T polymorphism was done by the polymerase chain reaction-restriction fragment length polymorphism (PCR-RFLP). ${ }^{[9]}$ The amplification reaction was carried out using primers: 5'-GGAGACTATTCAGAAGCTAA-3' and 5'-GAAGAGCCTGAAGACTGACT-3' and the following cycling conditions. Each amplification reaction was performed with $100 \mathrm{ng}$ of genomic DNA; 10 pmol of each primer; $2 \mu \mathrm{L}$ of $10 \times$ buffer solution; $200 \mu \mathrm{mol} / \mathrm{L}$ each of dATP, dCTP, dGTP, and dTTP; and $1 \mathrm{U}$ of Taq polymerase in a total volume of $20 \mu \mathrm{L}$. The DNA fragments were generated from initial denaturation at $95^{\circ} \mathrm{C}$ for 2.5 minutes, followed by 30 cycles of denaturation at $94^{\circ} \mathrm{C}$ for 30 seconds, annealing at $58^{\circ} \mathrm{C}$ for 40 seconds, and extension at $72^{\circ} \mathrm{C}$ for 60 seconds, with final extension at $72^{\circ} \mathrm{C}$ for 5 minutes. The amplification products were digested with the restriction enzyme XbaI, and the fragments were separated on a $4 \%$ agarose gel. Five randomly chosen samples with positive genotyping results were confirmed further by direct sequencing of the PCR products with an automated DNA sequencer.

Statistical analyses were performed using SPSS 14.0. The mean levels of TC, TG, LDL, and HDL in different groups were compared using Student's $t$-test. Frequencies of the genotypes and alleles were analyzed using 
the $\chi^{2}$ test. The odds ratio (OR) and 95\% confidential intervel (CI) were calculated to measure the strength of the association. Statistical significance was defined as $P<0.05$.

\section{Results}

\section{Clinical characteristics}

The characteristics of study subjects, patients and controls are summarized in Table 1. The two groups were well matched for age and sex. There were no significant differences in BMI, smoking history or history

Table 1: Clinical characteristic of case patients and control subjects

\begin{tabular}{lcc}
\hline & $\begin{array}{c}\text { Patients } \\
(\mathrm{n}=130)\end{array}$ & $\begin{array}{c}\text { Controls } \\
(\mathrm{n}=100)\end{array}$ \\
\hline Age (mean) & $56.72 \pm 11.35$ & $53.82 \pm 13.26$ \\
Male sex,\% (M/F) & $57.6(60 / 70)$ & $53.0(53 / 47)$ \\
BMI & $23.8 \pm 2.2$ & $23.9 \pm 3.1$ \\
Smokers, \% (n) & $9.2(12)$ & $8.0(8)$ \\
History of hypertension, \% (n) & $47.7(62)^{*}$ & $4.0(4)$ \\
History of diabetes, \% (n) & $20.0(26)^{*}$ & $3.0(3)$ \\
History of ischemic heart & $4.6(6)$ & $2.0(2)$ \\
disease, \% (n) & & \\
TC, mmol/L & $4.56 \pm 1.08^{\#}$ & $4.23 \pm 0.34$ \\
TG, mmol/L & $1.83 \pm 0.57^{\#}$ & $1.19 \pm 0.15$ \\
HDL - cholesterol, mmol/L & $1.34 \pm 0.83^{\#}$ & $1.44 \pm 0.99$ \\
LDL - cholesterol, mmol/L & $2.75 \pm 1.00^{\#}$ & $2.18 \pm 0.63$ \\
Family history of Cl & $36.2(47)^{*}$ & $0(0)$ \\
\hline
\end{tabular}

${ }^{*} P<0.01$ for the comparison between patients and controls: ${ }^{\#} P<0.05$ for the comparison between patients and controls but more significant difference could be seen in TC, TG, HDL-C, and LDL-C between the two groups; $\mathrm{Cl}$ : Cerebral infarction

Table 2: Genotype and allele frequencies of ApoB C7673T polymorphism in different groups

\begin{tabular}{lccccc}
\hline \multirow{2}{*}{ Group } & \multicolumn{2}{c}{ Genotype $\mathrm{n}(\%)$} & & \multicolumn{2}{c}{ Allele frequency } \\
\cline { 2 - 3 } \cline { 6 - 6 } & CC & TC or TT & & T & C \\
\hline CIFH $(\mathrm{n}=47)$ & $31(66.0)$ & $16(34.0)$ & & $0.170^{*}$ & 0.83 \\
CINFH $(\mathrm{n}=83)$ & $68(81.9)$ & $15(18.1)$ & & $0.09^{*}$ & 0.91 \\
Control $(\mathrm{n}=100)$ & $93(93.0)$ & $7(7.0)$ & & 0.04 & 0.96 \\
\hline
\end{tabular}

For comparison with the control group, ${ }^{*} \chi^{2}=12.78, P<0.01$; OR $=4.92$, $95 \% \mathrm{Cl}[1.89 \sim 13.15] ; \mathrm{RR}=4.26,95 \% \mathrm{Cl}\left[1.90 \sim 9.59\right.$ ]; $\# \chi^{2}=3.91$, $P<0.05 ; \mathrm{OR}=2.38,95 \% \mathrm{Cl}[0.92 \sim 6.32] ; \mathrm{RR}=2.29,95 \% \mathrm{Cl}[1.0 \sim 5.26]$; $\mathrm{CIFH}$ - Cerebral infarction with family history; CINFH - Cerebral infarction with no family history of ischemic heart diseases. The difference in history of hypertension, history of diabetes and family history of CI was significant.

\section{Allele frequencies of apolipoprotein B gene C7673T polymorphism and genotypes}

Results of the C7673T polymorphism are shown in Figure 1 and the genotype distribution and the allele frequency for APOB C7673T polymorphism in Table 2. Genotype frequencies did not significantly deviate from Hardy-Weinberg equilibrium in patients with $\mathrm{CI}$ and in controls $(P>0.05)$. As shown in Table 2 , there were only two genotypes (TC and CC) in CIFH and CINFH groups, while there were three genotypes (TC, CC, and TT) in the control group. The T alleles frequency of the C7673T polymorphism was significantly higher in CIFH and CINFH groups when compared with the control group $(P<0.01 ; P<0.05 ;$ respectively $)$. And the $\mathrm{T}$ allele frequency in CIFH group (0.17) was higher than CINFH (0.09) group $(P=0.056)$, but with no statistical significant difference.

\section{Effect on lipid profile}

The relation between the C7673T polymorphism and the serum lipids in CIFH, CINFH and the control groups are shown in Table 3. In both the CIFH and CINFH groups the serum levels of TC and LDL cholesterol were significantly high in the TT and TC genotypes than that in the CC genotype $(P<0.01)$, while the serum level of HDL cholesterol in the TT and TC genotypes

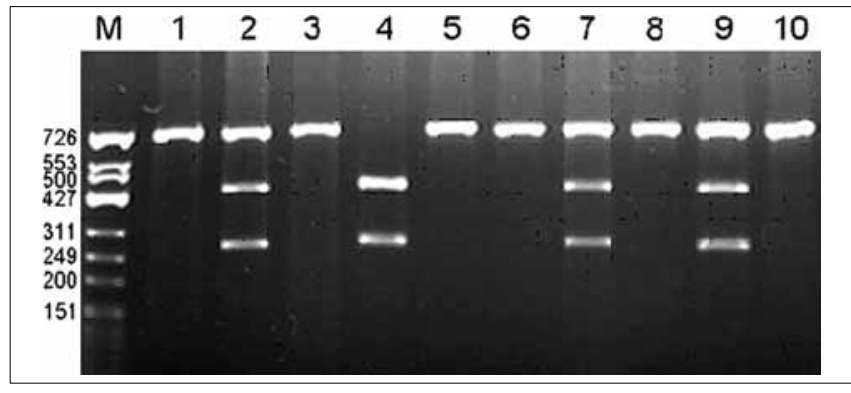

Figure 1: Restriction fragment length polymorphism electrophoretogram for the C7673T polymorphism of apolipoprotein B gene. Lanes 1,3,5: Genotype CC; Lanes 4: Genotype TT; Lanes 2: Genotype TC

\begin{tabular}{|c|c|c|c|c|c|c|c|c|c|}
\hline Group & Genotype & $\mathrm{n}$ & $\begin{array}{c}\text { TC } \\
\mathrm{mmol} / \mathrm{l}\end{array}$ & $\begin{array}{c}\text { TG } \\
\mathrm{mmol} / \mathrm{l}\end{array}$ & $\begin{array}{c}\text { LDL-C } \\
\mathrm{mmol} / \mathrm{l}\end{array}$ & $\begin{array}{l}\mathrm{HDL}-\mathrm{C} \\
\mathrm{mmol} / \mathrm{l}\end{array}$ & $\begin{array}{c}\text { ApoAl } \\
\mathrm{mmol} / \mathrm{l}\end{array}$ & $\begin{array}{c}\text { ApoB } \\
\mathrm{mmol} / \mathrm{l}\end{array}$ & $\begin{array}{c}\mathrm{Lp}(\mathrm{a}) \\
\mathrm{mmol} / \mathrm{l}\end{array}$ \\
\hline \multirow[t]{3}{*}{$\mathrm{CIFH}$} & $\mathrm{CC}$ & 31 & $4.56 \pm 1.48$ & $1.82 \pm 0.57$ & $2.55 \pm 1.31$ & $1.35 \pm 1.29$ & $1.44 \pm 0.25$ & $1.17 \pm 0.72$ & $0.25 \pm 0.16$ \\
\hline & $\mathrm{TC}$ or TT & 16 & $5.02 \pm 0.45^{*}$ & $1.89 \pm 0.92$ & $3.07 \pm 1.04^{*}$ & $0.79 \pm 0.42^{*}$ & $1.25 \pm 0.22$ & $1.22 \pm 1.01$ & $0.32 \pm 0.18$ \\
\hline & Total & 47 & $4.58 \pm 1.42$ & $1.85 \pm 0.69$ & $2.72 \pm 1.35$ & $1.33 \pm 1.24$ & $1.42 \pm 0.25$ & $1.20 \pm 0.73$ & $0.27 \pm 0.15$ \\
\hline \multirow[t]{3}{*}{ CINFH } & CC & 68 & $4.50 \pm 0.86$ & $1.72 \pm 0.89$ & $2.63 \pm 0.85$ & $1.36 \pm 0.53$ & $1.35 \pm 0.33$ & $1.02 \pm 0.37$ & $0.22 \pm 0.10$ \\
\hline & TC or TT & 15 & $4.91 \pm 0.81^{*}$ & $1.81 \pm 0.53$ & $3.00 \pm 0.49^{*}$ & $1.11 \pm 0.74^{*}$ & $1.38 \pm 0.19$ & $0.98 \pm 0.61$ & $0.29 \pm 0.12$ \\
\hline & Total & 83 & $4.56 \pm 0.85$ & $1.75 \pm 0.87$ & $2.78 \pm 0.81$ & $1.35 \pm 0.56$ & $1.36 \pm 0.32$ & $1.01 \pm 0.39$ & $0.24 \pm 0.11$ \\
\hline \multirow[t]{3}{*}{ Control } & $\mathrm{CC}$ & 93 & $4.12 \pm 0.34$ & $1.19 \pm 0.16$ & $2.17 \pm 0.64$ & $1.46 \pm 1.06$ & $1.29 \pm 0.26$ & $0.79 \pm 0.23$ & $0.19 \pm 0.18$ \\
\hline & TC or TT & 7 & $4.57 \pm 0.33$ & $1.18 \pm 0.10$ & $2.27 \pm 0.63$ & $1.38 \pm 0.15$ & $1.46 \pm 0.31$ & $0.77 \pm 0.11$ & $0.21 \pm 0.13$ \\
\hline & Total & 100 & $4.23 \pm 0.34$ & $1.19 \pm 0.15$ & $2.18 \pm 0.63$ & $1.44 \pm 0.99$ & $1.31 \pm 0.27$ & $0.79 \pm 0.21$ & $0.19 \pm 0.15$ \\
\hline
\end{tabular}

For the comparison with the CC genotype, $P<0.05$; CIFH - Cerebral infarction with family history; CINFH - Cerebral infarction with no family history 
was significantly lower than that in the CC genotype $(P<0.05)$. There were no significant differences in the serum levels of TG, ApoAI, ApoB100 and Lp(a) $(P>0.05)$ among the subgroups in the CIFH, CINFH, and controls groups.

\section{Discussion}

Cerebral infarction is a disease with familial clustering ${ }^{[10,11]}$ and is associated with ApoB. ${ }^{[12]}$ The gene coding for $A$ poB is polymorphic and has a close relationship with the pathogenesis of atherosclerosis; $;{ }^{[13]}$ however, little is known about the role of the APOB polymorphism in patients with CIFH. In our study, the $\mathrm{T}$ allele frequency was significantly higher in patients with CIFH and CINFH than in control subjects $(P<0.01, P<0.05$, respectively). Further, the T allele frequency in the CIFH group (0.17) was higher than in the CINFH (0.09) group. These results suggest the $\mathrm{T}$ allele of APOB C7637T polymorphism was not only associated with cerebral infarction, but also with CIFH in Han Chinese from Changsha. In our earlier study we have shown an association of T allele of APOB C7673T in cerebral hemorrhage. ${ }^{[8]}$ The results from these two studies show further evidence for the association between atherosclerosis and APOB.

The APOB is located on chromosome $2 \mathrm{q} 23$, spanning approximately $43 \mathrm{~kb}$ and has 29 exons and 28 introns. ${ }^{[14]}$ Polymorphisms in APOB may be associated with increased or decreased levels of APOB and LDL cholesterol. ${ }^{[15,16]}$ The C7673T polymorphism has been located just adjacent to the area of the LDL receptor. This polymorphism is a consequence of a $\mathrm{C}$ to $\mathrm{T}$ transversion at nucleotide 7673 in exon 26, which converts the 2488 codon ACC to ACT. The C7673T mutation creates an $\mathrm{XbaI}$ recognition site, that is called allele $\mathrm{T}(\mathrm{X}+)$, digestion of the PCR product of the mutant allele by this enzyme generates 2 fragments ( 433 and $277 \mathrm{bp}$ ). Though polymorphism of APOB C7673T does not lead to direct amino acid change, it still may play an important role in the pathogenesis of atherosclerosis and is related to coronary heart disease and myocardial infarction. ${ }^{[7]}$ All this suggests that the C7673T polymorphism of ApoB should have a potential effect on the pathogenesis of CI. Our study suggests that the T allele of APOB C7637T polymorphism is associated with $\mathrm{CI}$ and $\mathrm{CIFH}$ in Han Chinese from Changsha.

Our study found the serum TC and LDL cholesterol levels in patients with TC or TT genotypes were significantly higher while the HDL cholesterol was significantly lower than those in CC genotype. Some of the earlier studies have shown that the APOB C7673T polymorphism was associated with the serum levels of TC, LDL cholesterol and ApoB, and played an important role in the pathogenesis of atherosclerosis. ${ }^{[12,17]}$ Saha ${ }^{[17]}$ demonstrated significantly low levels of serum HDL cholesterol in TC genotype than in the CC genotype, and the T allele of APOB C7673T polymorphism was negatively associated with the serum HDL cholesterol level. They also found that the median serum TC level in TT genotype was $11 \sim 14 \%$ higher than that in CC genotype of the C7673T polymorphism, and the median serum LDL cholesterol level in TT genotype was $21 \%$ higher than that in CC genotype. ${ }^{[17]}$ Our findings were similar to the observations in the earlier studies, ${ }^{[17,18]}$ and have further established the association between the APOB C7673T polymorphism serum lipid metabolism in Han Chinese from Changsha.

The in vivo catabolism of serum lipoprotein is by its interaction with the lipoprotein receptor. Lipoprotein receptor is the protein located on the cell membrane and has a high affinity for the serum lipoprotein and the receptors are located on all the cell membranes, particularly on the hepatic cells and involved in the catabolism of LDL, intermediate-density lipoprotein (IDL) and very low-density lipoprotein (VLDL) cholesterol. ${ }^{[19]}$ Renges et al., ${ }^{[20]}$ showed that APOB polymorphism was an important genetic marker, and exhibit transmission disequilibrium with other nearby DNA sequence mutations which could also affect serum lipid metabolism. Those DNA sequence mutations may occur in the ApoB receptor combination area, leading to $A$ poB protein structure changes and thus decreasing the ability of LDL cholesterol to bind to the receptor. This can affect the catabolism of LDL cholesterol and lead to increased serum lipid levels in vivo. ${ }^{[2]}$ So we suppose APOB C7673T polymorphism correlates with CI, especially CIFH. It may be an internal susceptibility factor of serum lipid level increasing, and by changing the metabolism of serum lipid in vivo indirectly, APOB C7673T polymorphism takes part in the progression of atherosclerosis, and consequently takes part in the onset and progression of $\mathrm{CI}$, especially of CIFH. Validation of our findings will require additional studies with more subjects.

\section{Acknowledgments}

This study is supported by grants from National Natural Science Foundation of China (30600199).

\section{References}

1. Wu L, Takahashi K, Kobayashi S, Tian G, Song L, Matui R, et al. Clinical characteristies of cerebral infarction in China and Japan. Rinsho Shinkeigaku 2004;44:335-41.

2. Shao YN, Shen J, Tan YF. A study on the etiology and epidemiology of familial aggregation in arteriosclerotic cerebral infarction. Zhonghua Liu Xing Bing Xue Za Zhi 1997;18:275-8. 
3. Tonk M, Haan J. A review of genetic causes of ischemic and hemorrhagic stroke. J Neurol Sci 2007;257:273-9.

4. Kalaria RN. Advances in molecular genetics and pathology of cerebrovascular disorders. Trends Neurosci 2001;24:392-400.

5. Young SG. Recent progress in understanding apolipoprotein B. Circulation 1990;82:1574-94.

6. Chen Z, Fitzgerald RL, Saffitz JE, Semenkovich CF, Schonfeld G. Amino terminal $38.9 \%$ of apolipoprotein B-100 is sufficient to support cholesterol-rich lipoprotein production and atherosclerosis. Arterioscler Thromb Vasc Biol 2003;23:668-74.

7. Baroni MG, Berni A, Romeo S, Arca M, Tesorio T, Sorropago G, et al. Genetic study of common variants at the Apo E, Apo AI, Apo CII, Apo B, lipoprotein lipase (LPL) and hepatic lipase (LIPC) genes and coronary artery disease (CAD): Variation in LIPC gene associates with clinical outcomes in patients with established CAD. BMC Med Genet 2003;4:8.

8. Hu, ZY, Zhang L, Yang QD. Effect of APoB polymorphism on plasma lipid levels and cerebral hemorrhage in Changsha Han Chinese.Zhong Nan Da Xue Xue Bao Yi Xue Ban[J]. 2008;33:494-9.

9. Lin SK, Kao JT, Tsai SM, Tsai LY, Lin MN, Lai CJ, et al. Association of apolipoprotein $\mathrm{E}$ genotypes with serum lipid profiles in a healthy population of Taiwan. Ann Clin Lab Sci 2004;34:443-8.

10. Jousilahti P, Rastenyte D, Tuomilehto J, Sarti C, Vartiainen E. Parental history of cardiovascular disease and risk of stroke. A prospective follow-up of 14371 middle-aged men and women in Finland. Stroke 1997;28:1361-6.

11. Meschia JF, Atkinson EJ, O'Brien PC, Brott TG, Brown RD, Jr., Hardy J. Familial clustering of stroke according to proband age at onset of presenting ischemic stroke. Stroke 2003;34:e89-91.

12. Benn M, Nordestgaard BG, Jensen JS, Tybjaerg-Hansen A.
Polymorphisms in apolipoprotein B and risk of ischemic stroke. J Clin Endocrinol Metab 2007;92:3611-7.

13. Panaviotou A, Griffin M, Georgiou N, Bond D, Tyllis T, Tziakouri-Shiakalli $\mathrm{C}$, et al. ApoB/ApoA1 ratio and subclinical atherosclerosis. Int Angiol 2008;27:74-80.

14. Whitfield AJ, Barrett PH, van Bockxmeer FM, Burnett JR. Lipid disorders and mutations in the APOB gene. Clin Chem 2004;50:1725-32.

15. Humphries SE. DNA polymorphisms of the apolipoprotein genes - their use in the investigation of the genetic component of hyperlipidaemia and atherosclerosis. Atherosclerosis 1988;72:89-108.

16. Bentzen J, Jorgensen T, Fenger M. The effect of six polymorphisms in the Apolipoprotein B gene on parameters of lipid metabolism in a Danish population. Clin Genet 2002;61:126-34.

17. Carmena R, Duriez P, Fruchart JC. Atherogenic lipoprotein particles in atherosclerosis. Circulation 2004;109:III2-7.

18. Saha N, Tay JS, Humphries SE. Apolipoprotein B-gene DNA polymorphisms (XbaI and EcoRI), serum lipids, and apolipoproteins in healthy Chinese. Genet Epidemiol 1992;9:1-10.

19. Bovenschen N, Mertens K, Hu L, Havekes LM, van Vlijmen BJ. LDI receptor cooperates with LDL receptor-related protein in regulating plasma levels of coagulation factor VIII in vivo. Blood 2005;106:906-12.

20. Renges HH, Wile DB, McKeigue PM, Marmot MG, Humphries SE Apolipoprotein B gene polymorphisms are associated with lipid levels in men of South Asian descent. Atherosclerosis 1991;91:267-75.

Accepted on 02-07-2009

Source of Support: Grants from National Natural Science Foundation of China (30600199), Conflict of Interest: None declared.

\section{Staying in touch with the journal}

1) Table of Contents (TOC) email alert Receive an email alert containing the TOC when a new complete issue of the journal is made available online. To register for TOC alerts go to www.neurologyindia.com/signup.asp.

\section{2) RSS feeds}

Really Simple Syndication (RSS) helps you to get alerts on new publication right on your desktop without going to the journal's website. You need a software (e.g. RSSReader, Feed Demon, FeedReader, My Yahoo!, NewsGator and NewzCrawler) to get advantage of this tool. RSS feeds can also be read through FireFox or Microsoft Outlook 2007. Once any of these small (and mostly free) software is installed, add www.neurologyindia.com/rssfeed.asp as one of the feeds. 